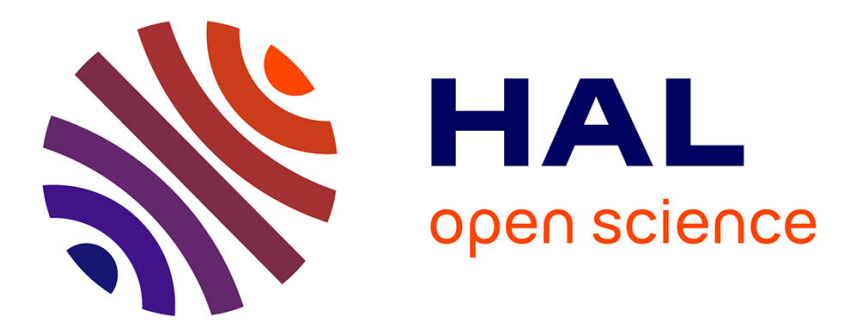

\title{
Inverse identification of the acoustic pressure inside a U-shaped pipe line based on acceleration measurements
}

Romain Beauvais, Adrien Pelat, François Gautier, Joel Gilbert

\section{To cite this version:}

Romain Beauvais, Adrien Pelat, François Gautier, Joel Gilbert. Inverse identification of the acoustic pressure inside a U-shaped pipe line based on acceleration measurements. Mechanical Systems and Signal Processing, 2021. hal-03370812

\section{HAL Id: hal-03370812 \\ https://hal.science/hal-03370812}

Submitted on 8 Oct 2021

HAL is a multi-disciplinary open access archive for the deposit and dissemination of scientific research documents, whether they are published or not. The documents may come from teaching and research institutions in France or abroad, or from public or private research centers.
L'archive ouverte pluridisciplinaire HAL, est destinée au dépôt et à la diffusion de documents scientifiques de niveau recherche, publiés ou non, émanant des établissements d'enseignement et de recherche français ou étrangers, des laboratoires publics ou privés. 


\title{
Inverse identification of the acoustic pressure inside a U-shaped pipe line based on acceleration measurements
}

\author{
Romain Beauvais $^{(a)(b)}$, Adrien Pelat ${ }^{(b)}$, François Gautier ${ }^{(b)}$, Joël Gilbert ${ }^{(b)}$, \\ Véronique Florquin ${ }^{(a)}$, Guillaume Vandenbossche ${ }^{(a)}$ \\ (a) SIM Engineering, 26 Rue du Président Paul Doumer, 59650 Villeneuve-d'Ascq, \\ FRANCE \\ (b) Laboratoire d'Acoustique de l'Université du Mans, UMR CNRS 6613, Avenue Olivier \\ Messiaen, 72085 Le Mans, FRANCE
}

In Oil \& Gas industry, the high internal sound pressure levels in pipe lines often cause significant structural vibrations. Quantification of the internal acoustic pressures is therefore an important step in understanding the global fluid-structure behaviour of such systems. However, direct acoustic measurements are often not possible since they generally require intrusive access to the pipe interior. In this paper an inverse procedure is proposed to identify the internal sound pressure field within the bend of a U-shaped pipe, from non-intrusive acceleration measurements on the mechanical structure. First, the accelerance transfer functions are measured in the bends and synthesized using a modal expansion. Under operational excitation conditions, the internal sound pressure is then obtained from a Tikhonov regularization of the inverse problem. The limits of the methodology are investigated numerically and experimentally to serve as a fast diagnostic tool for in situ vibration treatment of piping systems.

Keywords: fluid-structure coupling, Unbalanced forces, Modal analysis, Regularization method, Inverse problem, Non-intrusive measurement. 


\section{Introduction}

In the context of Oil \& Gas industry, gases are conveyed along pipe lines using compressors that act as acoustic sources. The induced acoustic pulsations lead to mechanical vibrations due to the fluid-structure coupling between the gas column and the pipe. The amplitude of such vibrations can occasionally threaten the safety of an industrial installation, because of either undersized pipe supports, or high sound pressure levels due to acoustic resonances along the pipe (Tison \& al [1], Nakamura [2]).

To treat these problems, in-situ diagnostic studies known as "troubleshooting piping systems", require access to both sound pressure and mechanical acceleration measurements. However, direct sound pressure measurements are rarely possible for safety reasons, such as volatility of the internal fluid, and the extreme experimental conditions (measurement points not accessible, large thermal and pressure variations). Formulation of an inverse solution is then relevant, since it potentially provides the sound pressure in the internal fluid from mechanical vibration measurement in a non intrusive way.

The use of inverse methods to reconstruct the internal pressure field by measuring wall displacements, is a recurrent theme in the literature, especially in problems of aeroacoustics and aerodynamics applied to aircraft wings. For certain applications, the aim is to determine in real time the applied loads, stresses, and displacements for in-flight structural health monitoring (Shkarayev \& al [3]). Other authors use similar technics to get simultaneous aeroacoustic and aerodynamic measurements on a wing or a membrane, non-intrusively and with high precision (Stanford \& al [4], Carpenter \& Albertani [5], Brown \& al [6]). The subject is also treated in a more theoretical way to be extended to various problems in industry (Engl \& Kügler [7]). However, among all these cases, the complexity of the fluid velocity profiles in the vicinity of the studied surfaces, implies a very complex experimental measurement bench as well as highly elaborate implementation procedures.

Other similar approaches relating the acceleration field of a piping line to the internal pressure field, have also been developed to simulate the fluidstructure interaction in pipes. These models allow weak or strong couplings to be considered above the relevant acoustical cut-off frequencies. Such calculations are based on the use of complex analytical models (Wiggert \& al 
[8], Lavooij \& Tijsseling [9], Zhang \& Tijsseling [10]), or numerical models (Maess \& Lothar [11], Herrmann \& al [12]). The fluid-structure interaction is also studied in different pipe configurations, such as straight or L-shaped pipes (Li \& al [13]). All these methods require prior knowledge of certain physical parameters, such as boundary conditions, which can be difficult to estimate precisely.

The industrial gas piping systems which concern the application cases of the paper, involve weak couplings associated with light fluids. In this context, a quick and easy to set up experimental procedure, requiring only a few measuring points, is needed to estimate the fluid-structure interaction. Such procedure can be set up as long as the studied frequency range contains the relevant pipe bending modes, and the maximum frequency of interest is lower than the first acoustic cut-off frequency (plane wave assumption).These conditions are pertinent since the source (a reciprocating compressor) generates low frequency components (below $300 \mathrm{~Hz}$ ). For the first condition, the diameters of the pipes (between 50 and $300 \mathrm{~mm}$ ) are small compared to their length so the modes are assimilated to beam bending modes. For the second one, the gases inside the pipes can be very different from one study to another, with various temperatures, so the celerity can differ from $200 \mathrm{~m} . \mathrm{s}^{-1}$ to $1200 \mathrm{~m} \cdot \mathrm{s}^{-1}$. The cut-off frequency is then at least at $390 \mathrm{~Hz}$, which is above the frequency range of the waves generated by reciprocating compressors.

The main purpose of the approach is to identify the origin of high amplitude vibrations in an installation. A typical modal analysis of the structure indicates the frequencies for which these vibrations are likely to be annoying. However, in troubleshooting piping systems this information is very insufficient. The existence of excitation forces (so-called unbalances forces) due to wave propagation as well as of their resonant character is also a necessary condition to detect the appearance of important vibrations. Due to time, material and cost constraints, in-situ vibration studies require an experimental diagnostic tool light and quick to use to identify these acoustic unbalanced efforts. The reduction of complexity imply the use of a model driven by experimental data requiring a minimum of measuring points, considered modes, computation time and implementation procedure.

The present procedure is suitable for in-situ application, since it is: non intrusive, because the vibration measurement is carried out on the external sides of the pipes; fully experimental, because the mechanical modal param- 
eters of the pipes are extracted by an impact testing procedure; quick to set up, because a limited number of acceleration measurements at strategic points allows a faithful reconstruction of the internal pressure; light and low cost, as it requires the use of only one impact hammer and one accelerometer.

This procedure is tested on a U-shaped pipe composed of straight pipes and $90^{\circ}$ elbows (fig.1), where the pressure discrepancies in amplitude and phase between the elbows lead to an unbalancing of the forces applied to them, inducing vibrations (Blodgett [14]). The pipe diameter is $44 \mathrm{~mm}$ $\left(f_{c}=2264 \mathrm{~Hz}\right)$. Then the mechanical bending modes involved are much lower than the cut-off frequencies, which satisfy the plane wave hypothesis.

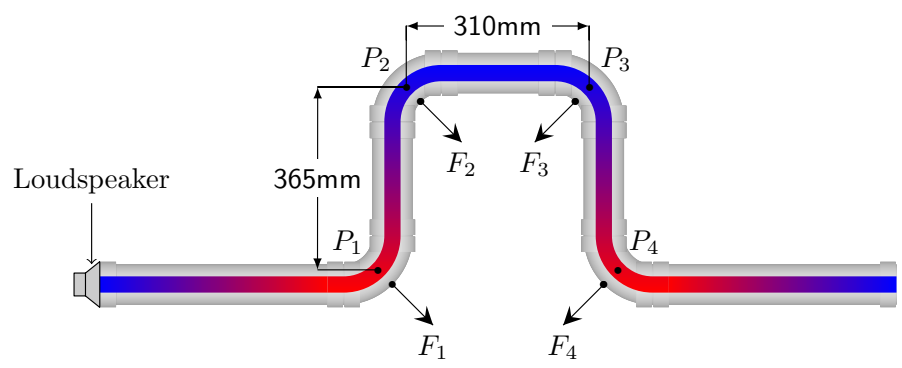

Figure 1: Schematic representation of the U-shaped pipe line, the internal acoustic pressure and the unbalanced forces applied at the elbows. Red and blue colors represent maximum and minimum acoustic pressure levels respectively.

This paper is organized into two parts. The first part deals with theoretical modelling of a fluid-structure coupling in a piping lines such as the one illustrated in the figure 1. For this purpose, the forces resulting from the internal pressure distribution are computed assuming a plane wave hypothesis. Incorporating these forces into the monodimensional equation of motion, leads to a relationship between acoustic pressure and mechanical acceleration. This introduces the direct problem (estimation of acceleration from pressure) and the inverse problem (estimation of pressure as a function of acceleration). Concerning the inverse problem, a regularization method is applied to ensure the stability of the calculation. The second part deals with the implementation of the theoretical model. In a preliminary phase, the modal basis of the U-shaped pipe is obtained from a modal expansion performed on accelerance measurements. The requirement for proper regularization of the inverse problem is demonstrated using a simulated case 
study, to estimate the impact of measurement noise on the stability of the inverse solution. Then experimental studies are conducted. The direct and inverse method are then tested on experimental signals obtained with a swept sine acoustic source provided by a loudspeaker. The aim is to evaluate the limits of the fluid-structure model using only four measurement points. Indeed, the fewer measurements are required, the more applicable the method is for in situ vibration diagnosis. Finally, an operational electro-pneumatic source, similar to a reciprocating compressor source, is connected to the inlet of the pipe to demonstrate the efficiency of the procedure for identifying the various factors involved in the case of strong mechanical vibrations. Such factors include acoustic resonance, acoustic components generated by the source or high flexibility of the pipe.

\section{Modeling of the fluid-structure coupling}

Modelling the fluid-structure coupling between the fluid and the structure requires an understanding of how forces on the U-shaped pipe are induced by the internal acoustic pressure.

\subsection{Description of the forces applied to the pipe line}

When a plane wave propagates into a bent pipe line, resulting forces appear at the bends. For long wavelengths in comparison to the length of each bend, the Ostrograsky's theorem can be applied (Kaltz [15]) which means that a uniform pressure distribution acting on any closed surface $S_{\text {tot }}$ leads to a zero resulting force:

$$
\int_{S_{\mathrm{tot}}} P \overrightarrow{d S}=\overrightarrow{0}
$$

As a consequence, by considering the surface $S_{\text {tot }}$ as the association of a lateral surface $S_{p}$, an input surface $S_{\text {in }}$ and an output surface $S_{\text {out }}$ (see fig.2, we get:

$$
\vec{F}=\int_{S_{p}} P \overrightarrow{d S}=-P S_{\text {in }} \vec{n}_{\text {in }}-P S_{\text {out }} \vec{n}_{\text {out }}
$$

with $\vec{n}_{\text {in }}$ and $\vec{n}_{\text {out }}$ being respectively the outgoing unit vectors located on the $S_{\text {in }}$ input and $S_{\text {out }}$ output sections (fig.2). Then if $S=S_{\text {in }}=S_{\text {out }}$, the force 
will be collinear to the bisector of the curvature angle of the elbow and along the outgoing normal $\vec{n}$ (where $\vec{n}_{\text {in }}+\vec{n}_{\text {out }}=-2 \sin (\theta / 2) \vec{n}$ ):

$$
\vec{F}=2 P S \sin \left(\frac{\theta}{2}\right) \vec{n}
$$

In the studied case, the experimental setup (fig.1) only involves perpendicular elbows $(\theta=\pi / 2)$, this corresponds to the most cases encountered in industrial applications. Then the resulting force applied to an $i^{\text {th }}$ elbow are:

$$
\vec{F}_{i}=P S_{i} \sqrt{2} \overrightarrow{n_{i}}
$$

where $i=[1,2,3,4]$ correspond to the index of each.

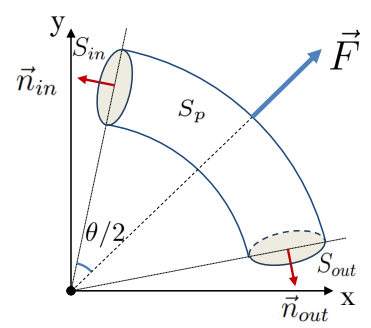

Figure 2: Scheme of the elbow and notations.

Depending on the geometry of the pipe and the distribution of the acoustic pressure field, these forces may not cancel each other out and lead to vibratory excitation, called "unbalanced forces" or "shaking forces" in the literature (Blodgett [14], Tison \& al [1]). As a consequence, the straight pipe segment lying between elbow $i$ and elbow $i+1$ is subject to the force:

$$
\Delta \vec{F}_{j}=S_{i+1} P_{i+1} \cdot \vec{n}_{\mathrm{out}, j}-S_{i} P_{i} \cdot \vec{n}_{\mathrm{in}, j}
$$

with $j=1$ to 3 , the straight segment pipe index, $S_{i}$ and $P_{i}$, the section and the pressure at the $i^{t h}$ elbow and $\vec{n}_{\text {in }, j}$ and $\vec{n}_{\text {out }, j}$, the outgoing unit vectors located respectively on the input and output section of the $j^{\text {th }}$ straight segment pipe. These vectors are collinear but oriented in opposite directions. 


\subsection{Direct problem: Response of a pipe line to internal pressure excitation}

As a preliminary step, the modal basis of the U-shaped pipe line is obtained from a modal fit of the accelerance measurements. From the discretization described above, the equations of motion of the pipe are written in matrix form as:

$$
[\mathbf{M}] \ddot{\mathbf{X}}+[\mathbf{C}] \dot{\mathbf{X}}+[\mathbf{K}] \mathbf{X}=\mathbf{F},
$$

with $\mathbf{F}=\left[F_{1}, \ldots, F_{N}\right]$ the $\mathrm{N} \times 1$ vector of the normal forces to the pipe wall, $N$ the number of degrees of freedom, $\mathbf{X}=\left[x_{1}, \ldots, x_{N}\right]$ the $\mathrm{N} \times 1$ vector of the displacements and $[\mathbf{M}],[\mathbf{C}]$ and $[\mathbf{K}]$ respectively the $\mathrm{N} \times \mathrm{N}$ mass, damping and stiffness matrices of the pipe.

Following the modal expansion principle, $\mathbf{X}$ can be expressed as:

$$
\mathbf{X}=[\boldsymbol{\Psi}] \mathbf{R}
$$

with $\mathbf{R}$ the $K \times 1$ vector of modal coordinates, where $K$ is the number of modes used, and $[\boldsymbol{\Psi}]=\left[\boldsymbol{\psi}_{1}|\ldots| \boldsymbol{\psi}_{K}\right]$ the $\mathrm{N} \times \mathrm{K}$ matrix of the mode shapes, each one satisfying:

$$
-\omega_{k}^{2} m_{k} \boldsymbol{\psi}_{k}+k_{k} \boldsymbol{\psi}_{k}=0
$$

Setting $[\boldsymbol{\Psi}]^{t}[\mathbf{M}][\boldsymbol{\Psi}]=\operatorname{diag}\left(m_{k}\right), \quad[\boldsymbol{\Psi}]^{t}[\mathbf{C}][\boldsymbol{\Psi}]=\operatorname{diag}\left(c_{k}\right), \quad[\boldsymbol{\Psi}]^{t}[\mathbf{K}][\boldsymbol{\Psi}]=$ $\operatorname{diag}\left(k_{k}\right)$ and orthonormalizing the mode shapes $\boldsymbol{\phi}_{k}=\boldsymbol{\psi}_{n, k} / \sqrt{m_{k}}$, the substitution of equation (7) in equation (6) and the projection on the modes $\phi_{k}$ leads to:

$$
[\mathbf{I}] \ddot{\mathbf{R}}+\operatorname{diag}\left(2 \xi_{k} \omega_{k}\right) \dot{\mathbf{R}}+\operatorname{diag}\left(\omega_{k}^{2}\right) \mathbf{R}=[\boldsymbol{\Phi}]^{t} \mathbf{F},
$$

where $\omega_{k}=\sqrt{\frac{k_{k}}{m_{k}}}$ and $\xi_{k}=\frac{c_{k}}{2 \sqrt{k_{k} m_{k}}}$ from the eigenvalues $\lambda_{k}=\omega_{k}\left(-\xi_{k}+\right.$ $\left.j \sqrt{1-\xi_{k}^{2}}\right)$.

Finally, assuming an harmonic excitation $\left(\mathbf{R}=\tilde{\mathbf{R}} e^{j \omega t}\right)$ in equation (9), the vector of accelerations $\left(\mathbf{a}=\ddot{\mathbf{X}}=-\omega^{2} \mathbf{X}\right)$ satisfying the equation of motion is:

$$
\mathbf{a}=-\omega^{2}[\boldsymbol{\Phi}][\mathbf{H}][\mathbf{\Phi}]^{t} \mathbf{F}=[\mathcal{H}] \mathbf{F}
$$

with

$$
[\mathbf{H}]=\operatorname{diag}\left(\frac{1}{\omega_{k}^{2}+2 j \xi_{k} \omega_{k} \omega-\omega^{2}}\right)
$$


2.3. Inverse problem: Identification of the internal acoustic pressure from mechanical measurements of pipe line vibrations

From the above model, an inverse procedure can be developed to estimate the internal acoustic pressure field resulting from mechanical measurements of the pipe line acceleration. Beginning with equation (10), the naive solution of the inverse problem is:

$$
\mathbf{F}(\omega)=[\mathcal{H}]^{-1} \mathbf{a}(\omega) .
$$

In an ideal noiseless case, this naive inversion makes it possible to identify the excitatory forces $(\mathbf{F})$ without special precautions. In the practical case of noisy acceleration data (a), a solution for the force may still be obtained, but with large errors, even for a favourable signal-to-noise ratio. Therefore, the procedure requires careful regularization in order to avoid instabilities during the inversion of the matrix $\mathcal{H}$ (Idier [16]).

The Tikhonov method is applied to deal with the regularization (Tikhonov [17]). The estimation, or residual, error $\varepsilon$ is defined by:

$$
\|[\mathcal{H}] \mathbf{F}-\mathbf{a}\|^{2}=\boldsymbol{\varepsilon} .
$$

An optimization procedure is applied to minimize this error. It consists of finding the values of $\mathbf{F}$ for which

$$
\frac{\partial \mathbf{J}_{\beta}}{\partial \mathbf{F}}=0,
$$

considering that

$$
\mathbf{J}_{\beta}=\|[\boldsymbol{H}] \mathbf{F}-\mathbf{a}\|^{2}+\beta\|\mathbf{F}\|^{2},
$$

with $\beta\|\mathbf{F}\|^{2}$ the adjustment term. The solution of equation (14) leads to the following approximation for $\mathbf{F}$ :

$$
\tilde{\mathbf{F}}=\left([\mathcal{H}]^{T}[\mathcal{H}]+\beta[\mathbf{I}]\right)^{-1}[\mathcal{H}]^{T} \mathbf{a} .
$$

The higher the $\beta$ value, the more the stability is ensured but the more the solution is altered. This means that there exists an optimal value of $\beta$, which can be determined by using the L-curve method (Hansen [18]). An example of this method is given in appendix A. In the following cases, the search for the optimal $\beta$ value is bounded between $10^{-6}$ and 1 . 


\section{Results}

This section describes the application of the direct and inverse methods described above to the specific case of a laboratory demonstrator of a Ushaped pipe.

\subsection{The experimental setup}

The experimental setup (fig.3) has been designed so that the involved modes are in the frequency range of interest of the in-situ reciprocating compressors (below $300 \mathrm{~Hz}$ ). In order to achieve this condition, according to pipe lengths possible to be implemented in the laboratory, the model was manufactured in PVC which makes it lighter but above all more flexible. It consists of a $44 \mathrm{~mm}$ inner diameter PVC pipe of $3.85 \mathrm{~m}$ full length (from the loudspeaker to the termination). This pipe contains 4 elbows separated by segments of lengths $0.365 \mathrm{~m}, 0.310 \mathrm{~m}$, and $0.375 \mathrm{~m}$ respectively (fig.1). Three different terminations are chosen: open, closed, or quasi anechoic (Dalmont \& Portier [19], see appendix B). These different terminations are used to obtain various sets of pressure fields inside the pipe in order to test the direct and inverse models with different acoustic configurations (section 3.4).

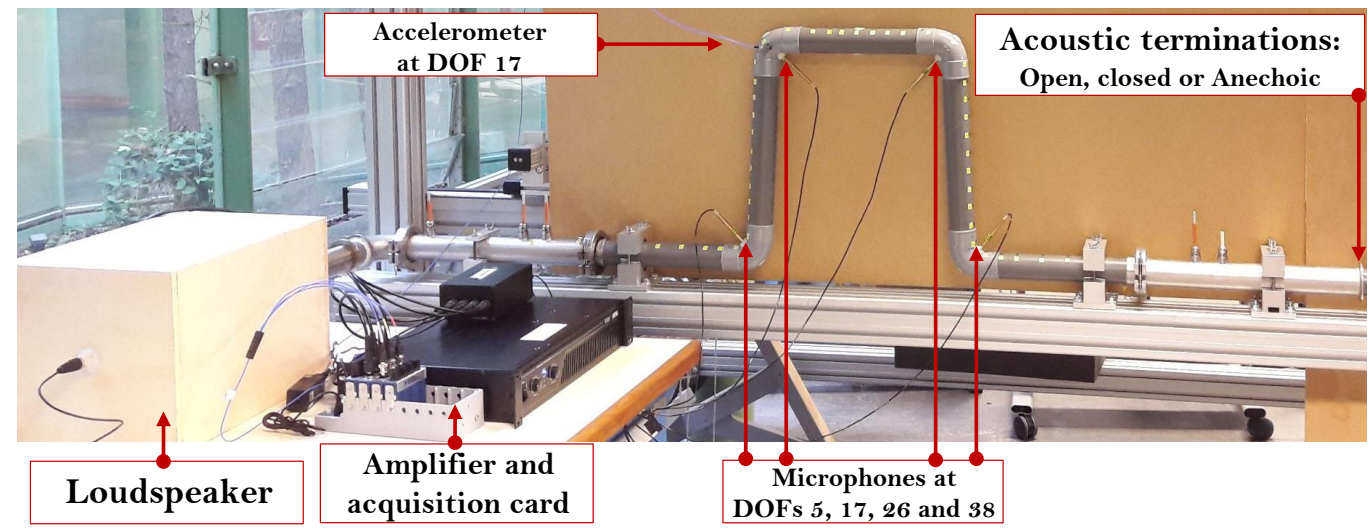

Figure 3: Experimental setup showing the pipe line, with arrows pointing to the various devices: The 4 microphones are respectively at the degrees of freedom $5,17,26,38$, and the accelerometer is at the degree of freedom 17.

The pipe is acoustically excited by a loudspeaker whose cut-off frequency is $40 \mathrm{~Hz}$. Acoustic pressure measurements inside the pipe are performed using 
1/4" G.R.A.S 40BP microphones located at each elbow, in conjunction with G.R.A.S Power Module Type 12AQ signal conditioners. Accelerations are measured using an ICP PCB 356A01 accelerometer of negligible mass that can be moved between various measurement points, corresponding to the yellow paths located along the U-shaped pipe (fig.3). The setup is controlled in Labview, with a National Instrument USB-4431 DAQ device.

\subsection{Modal analysis of the pipe}

The direct model presented in sec.2.2 and 2.3 requires prior identification of the modal parameters $f_{k}, \xi_{k}$ and $\phi_{k}$ for the pipe.

Experimental modal analysis is conducted in the low frequency range [0$250 \mathrm{~Hz}$ ] using an impact hammer (PCB 086D05) as the excitation source. This allows flexible tips to be used for measurements. The excitation level is then sufficient to obtain a completely satisfactory response (see fig.5). It was not necessary to carry out averages as these do not provide additional information. The analysis is carried out over 60 points along the pipe and 43 of them are located between the clamped points of the U-shaped PVC pipe (see yellow patches in figure 3). For each impact, the applied force is measured and the acceleration is measured at the second elbow along the outgoing normal direction $\vec{n}$ (fig.2), corresponding to the $17^{\text {th }}$ degrees of freedom starting from the left of the pipe.

Two steps are involved in the modal identification procedure. The first step leads to the extraction of the modal frequency and damping ratios, using the high resolution technique ESPRIT (Ege \& al [20]). The second step leads to the estimation of the order of the model and to the extraction of the mode shapes (Paiva \& al [21]).

Following this procedure, 12 modes are identified in the range $[0-250] \mathrm{Hz}$, which are shown in figure 4. As expected, some modes correspond to global motions such as rotation around $x$ (mode 1 ) or around $z$ (mode 3 ) and translation along $y$ (mode 5) while others correspond to more complex motion such as twisting modes (e.g. mode 4) or breathing modes (e.g. mode 11). 


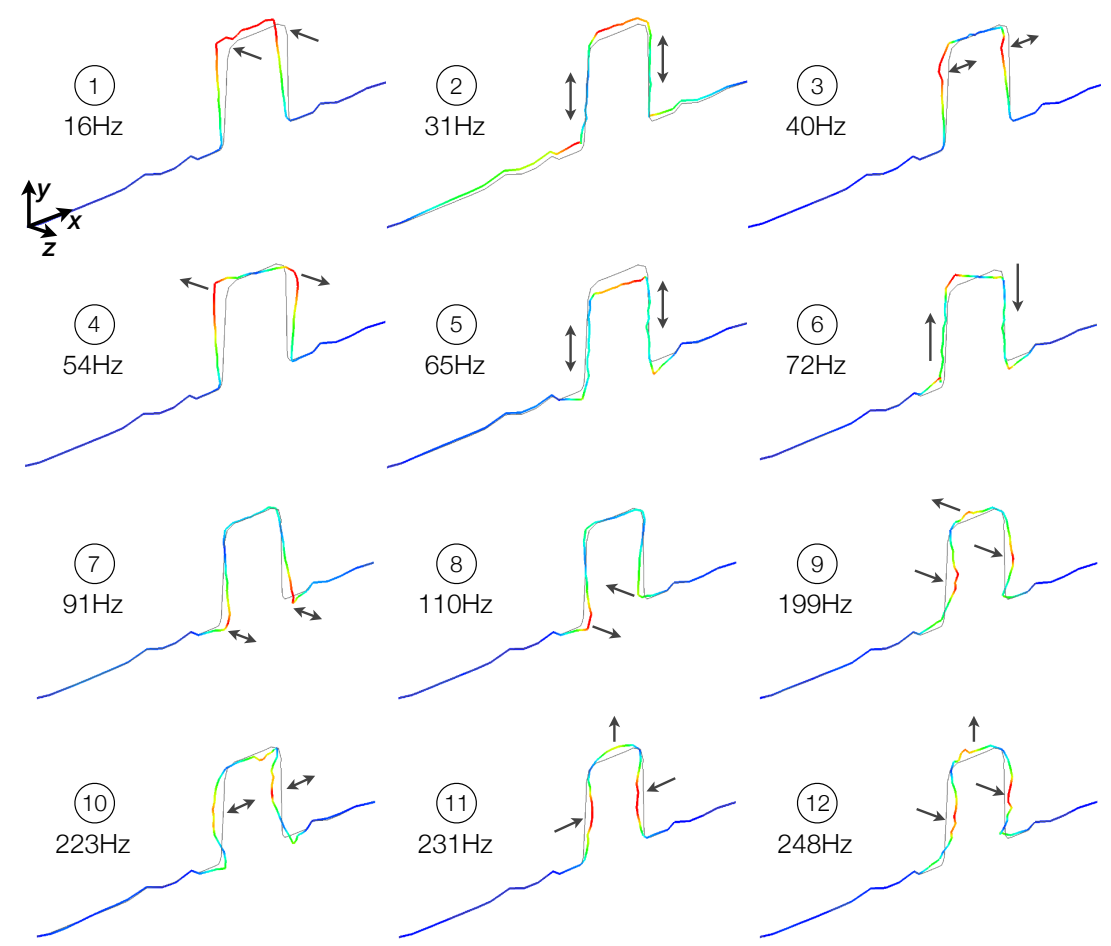

Figure 4: Mode shapes of the U-shaped pipe in the range [0-250] Hz.

More interestingly, these mode shapes can be assimilated to beam modes, for which each pipe section is considered as a point element. This assumption is justified by considering that the straight pipe are not being significantly deformed along their radial coordinates, which is verified in industrial installations. In addition, the resulting forces induced by the acoustical field are located only at the 4 elbows (eq.3).

Therefore the U-shaped pipe line is reasonably approximated by a discrete system with only 4 degrees of freedom, where each axis is collinear to the bisector of each elbow (fig.1).

In figure 3 , the four degrees of freedom correspond to the $5^{\text {th }}, 17^{\text {th }}, 26^{\text {th }}$ and $38^{\text {th }}$ yellow patches between the pipe clamped points. Thus, the following modal identification procedure is simply performed with only 4 impact testing points such that $[\mathcal{H}]$ is a $[4,4]$ matrix, involving 12 eigenvectors. This means that the initial continuous system has been simplified into a highly simplified discrete system $(\mathrm{N}=4$ and $\mathrm{K}=12)$, in accordance with the criteria for a 
quick and easy to use diagnostic tool.

The validity of the estimated modal parameters is verified by comparing synthesized and measured accelerance. Figure 5 shows very good agreement with the measurement for the co-localized accelerance $\mathcal{H}_{2,2}$ (at the second elbow), as well as for other accelerances (not shown here for the sake of conciseness). Therefore, the results of Figure 5 show that the experimental set-up is very well represented within the frequency band of interest [0-250] $\mathrm{Hz}$, using 12 modes.

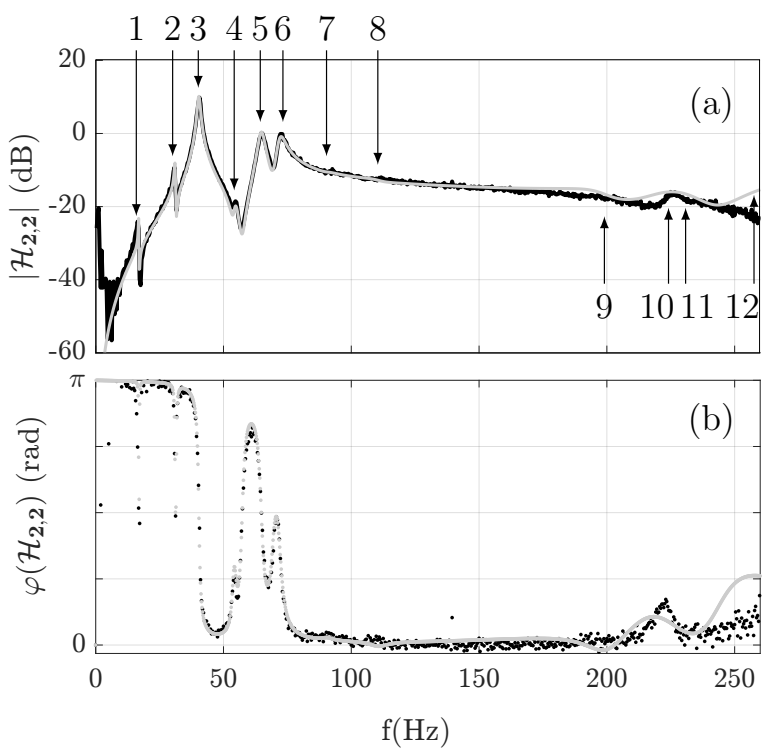

Figure 5: Measured (black) and synthesized (grey) accelerances along the outgoing normal $\vec{n}_{2}$ of elbow $2, \mathcal{H}_{2,2}=a_{2} / F_{2}$. (a): the module with the indicated frequencies location of the 12 extracted modes, the modes from 1 to 8 are the elbow flexion modes and the modes from 9 to 12 are the pipe flexion modes (fig.4). (b): the phase.

These modal parameters will subsequently be used in the transfer matrix of equations 10, 12 and 16 to solve respectively the direct, inverse non regularized and inverse regularized problems.

The relatively small number of modes and degrees of freedom is very attractive for simple and quick in situ characterization, in view of the indirect measurement of the internal pressure field. Note that due to the geometric imperfections and the small pipe diameters, the impact tests performed on the four elbows are not strictly collinear to the forces induced by the internal 
pressure during a fluid-structure coupling (i.e. in the X-Y plane).

\subsection{Estimation of the internal sound pressure from simulated data}

Based on previous analysis and before experimental applications, a preliminary evaluation of the efficiency of the regularization method is done when a controled noise is added to signals, following a simple simulation methodology (fig.6). First, simulated forces $\mathbf{F}$ are applied, from a given acoustic pressure field, to the 4 degrees of freedom of the structure. Then, using the modal parameters of the pipe obtained experimentally (part 3.2), the resolutions of the direct problem (eq.10) provide the acceleration a to which a noise $\varepsilon$ is added. Finally, the non-regularized or regularized inverse procedure (eq.12 or eq.16) is applied to the noisy signal in order to identify the force $\tilde{\mathbf{F}}$ and so the acoustic pressure.

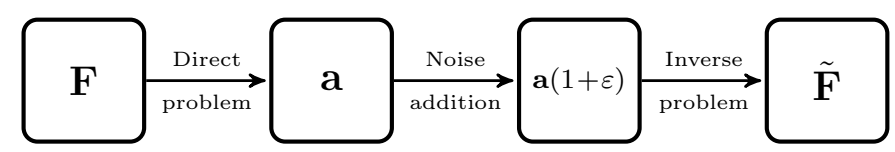

Figure 6: Methododology to estimate the impact of noise on the solution of the inverse problem (Eqs.12 and 16 for the unregulated and regulated solutions).

For this simulation, very noisy signals (like Sound Noise Ratios of $0 \mathrm{~dB}$ ) are used, but they are not representative of the in-situ reality, as they will not be considered satisfactory by the operator. The aim is only to demonstrate the robustness of Tikhonov's regularisation method for the present application.

For the present simulation, a white gaussian noise signal is considered in each elbow $(\mathbf{F})$. The $1^{\text {st }}$ line of the figure 7 , corresponds to the estimated acceleration at elbow 2, for which three different noise levels are added ( SNRs of $50 \mathrm{~dB}, 20 \mathrm{~dB}, 0 \mathrm{~dB}$, respectively in columns (a), (b) and (c)). The $2^{\text {nd }}$ line corresponds to the evolution of the regularization parameter $\beta$, for each configuration. An example of locating the optimal $\beta$ parameter from the L-curve method, at a given frequency and for different SNRs, is shown in appendix A (fig.A.1). In line 3, the input pressure (black line) in the elbow 2 is compared with the non-regularized (red line) and regularized (green line) solutions. As a reminder, the pressure is proportional to $\mathbf{F}$ (eq.4). 
As indicated in section 2, it appears that the higher the noise, the higher the optimal $\beta$ value and therefore the more the regularization is essential to ensure the consistency of the solution.
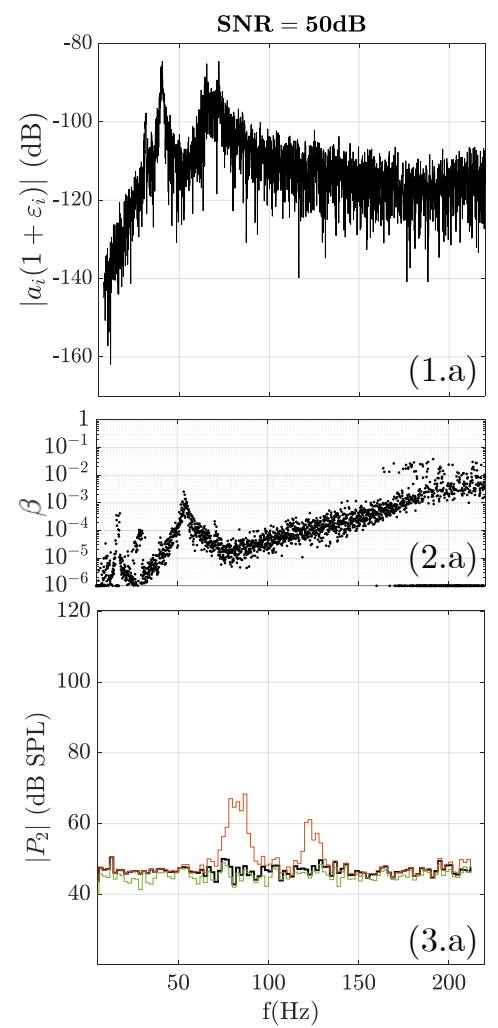
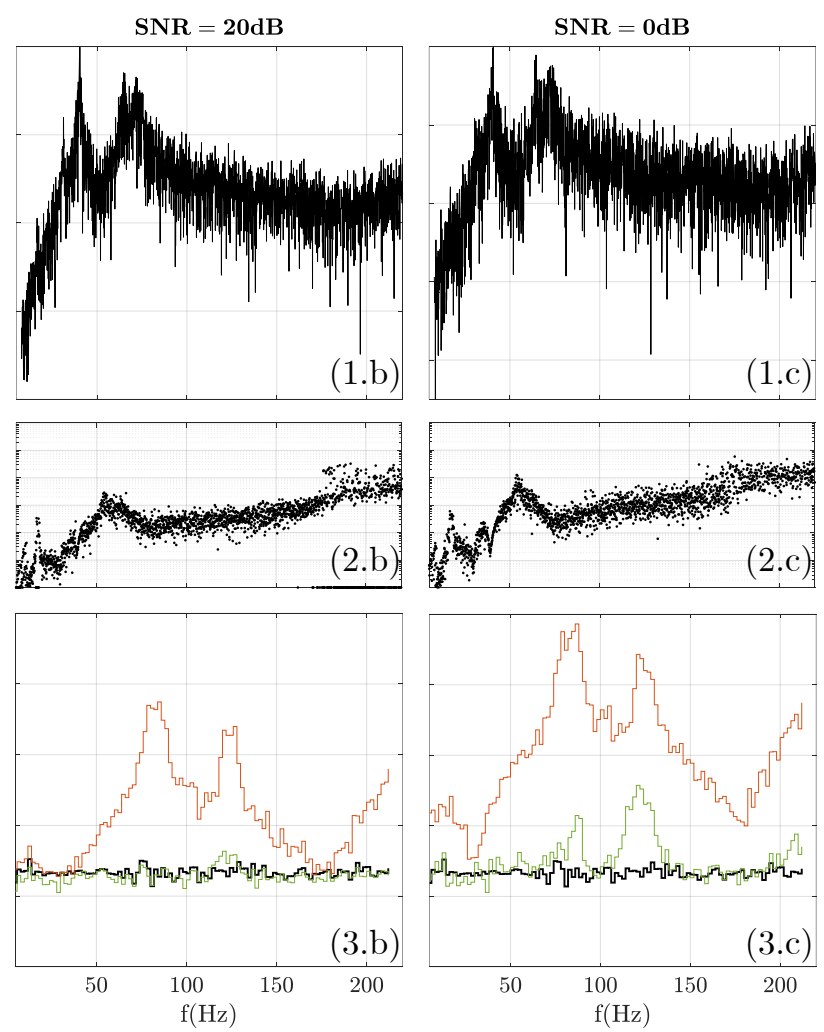
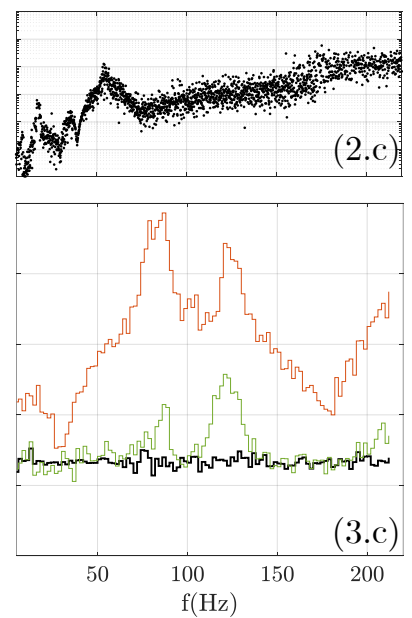

Figure 7: Simulation of the inverse method with acoustic gaussian white noise excitation (step 3 of figure 6) and 3 levels of added white noise, (a) SNR $=50 \mathrm{~dB}$, (b) SNR $=20 \mathrm{~dB}$, (c) SNR $=0$ dB. (1) Estimated acceleration spectra at the $2^{\text {nd }}$ elbow with added gaussian white noise (eq.10); (2) Regularisation parameter $\beta$ used for the inverse identification of the sound pressure (eq. 16); (3) Estimated sound pressure spectra obtained from the nonregularized (red) and regularized (green) inverse method (eqs. 12 and 16, respectively), compared with the initial input pressure (black).

To study more precisely the impact of regularization of the problem, the methodology described in figure 6 and presented in figure 7, is applied for a larger number of SNRs. It is then possible to evaluate the evolution of the error between the input and the output by gradually increasing the background noise $\varepsilon$ at the third step of the methodology. The error criterion is calculated as follows: 


$$
\epsilon=\frac{1}{4} \sum_{i=1}^{4} \frac{\int_{f_{1}}^{f_{2}}\left|\tilde{P}_{i}(f)-P_{i}(f)\right| \mathrm{d} f}{\int_{f_{1}}^{f_{2}} P_{i}(f) \mathrm{d} f},
$$

with $\tilde{\mathbf{P}}$ and $\mathbf{P}$, respectively the estimated and measured pressure vector in $\mathrm{dB}$ SPL, related to $\mathbf{F}$ (eq.4), the frequency band of interest is $f_{1}=10 \mathrm{~Hz}$ and $f_{2}=210 \mathrm{~Hz}$.

The comparison between the regularized and non-regularized solutions for the three added noise configurations demonstrates the usefulness of the regulation parameter $\beta$.

Note that when noise increases, the $\beta$ parameter becomes a more preeminent term in Tikhonov's relationship (eq.16) and the solution becomes very sensitive to it. However, $\beta$ is calculated numerically and is therefore not perfectly accurate. These small deviations have a slight effect on the solution, which can be seen in particular on a logarithmic scale. In the present case, between SNRs of $7.5 \mathrm{~dB}$ and $25 \mathrm{~dB}$, the solution is correctly reconstructed $(\epsilon$ $<1 \%$ ), but is oscillating according to the SNR. Then, below SNR of $5 \mathrm{~dB}$, the noise becomes too high and the solution is no longer correctly regularized $(\epsilon>2 \%)$.

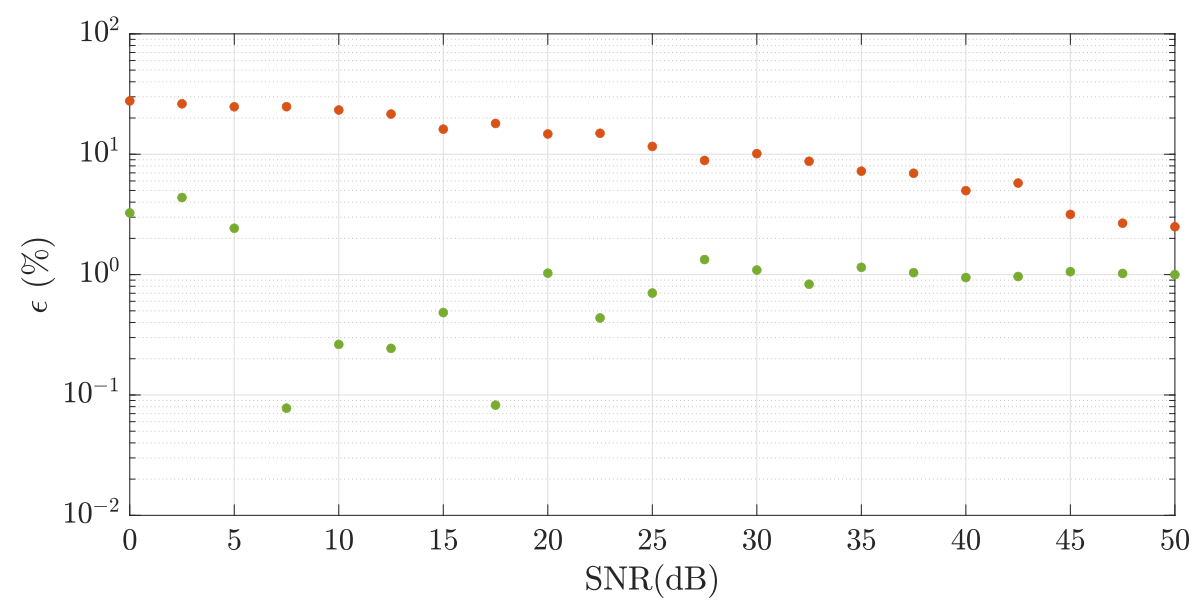

Figure 8: Error indicator (eq.17) between the input and output, using the methodology from the figure 6 , with regularization (green dots) and without regularization (red dots). 


\subsection{Experimental application: Internal sound pressure from swept sine ex- citation}

As a first experimental application, the case of the forced vibration response to an acoustic frequency swept sine in the range $[10,210] \mathrm{Hz}$ is considered. At the pipe inlet, acoustic excitation is generated by a loudspeaker, while at the outlet, anechoic, open and closed terminations are successively considered (fig.3). Sound pressure and acceleration are measured at each of the 4 elbows (along the outgoing normal $\vec{n}$ ). The pressure and acceleration measurements require averaging in order reduce the noise. In the present study, the averaging was performed on 10 consecutive measured signals of 10 seconds each.

The Figure 9 displays the acceleration at elbow 2 identified by the direct method (grey lines) for which the measured pressures are introduced into Eq. 10, with the three pipe terminations (anechoic, closed and opened). The identified accelerations are in very good agreement with the measurements (black lines). This experimentally validates the principle of describing the U-shaped pipe structure by only 4 degrees of freedom located at the elbows.

In the case of the anechoic termination (Figure 9(a)), the peaks correspond to resonances of the mechanical modes of the pipe that are excited by unbalanced forces due to acoustical phase shift between the elbows. In the case of the closed or open terminations (fig.9(b) and (c)), additional peaks appear and correspond to high amplitude forced excitation due to acoustic standing waves along the pipe. This suggest that in the case of coincidence between acoustical and mechanical modes, strong fluid-structure coupling can result in peaks of very high amplitudes. 

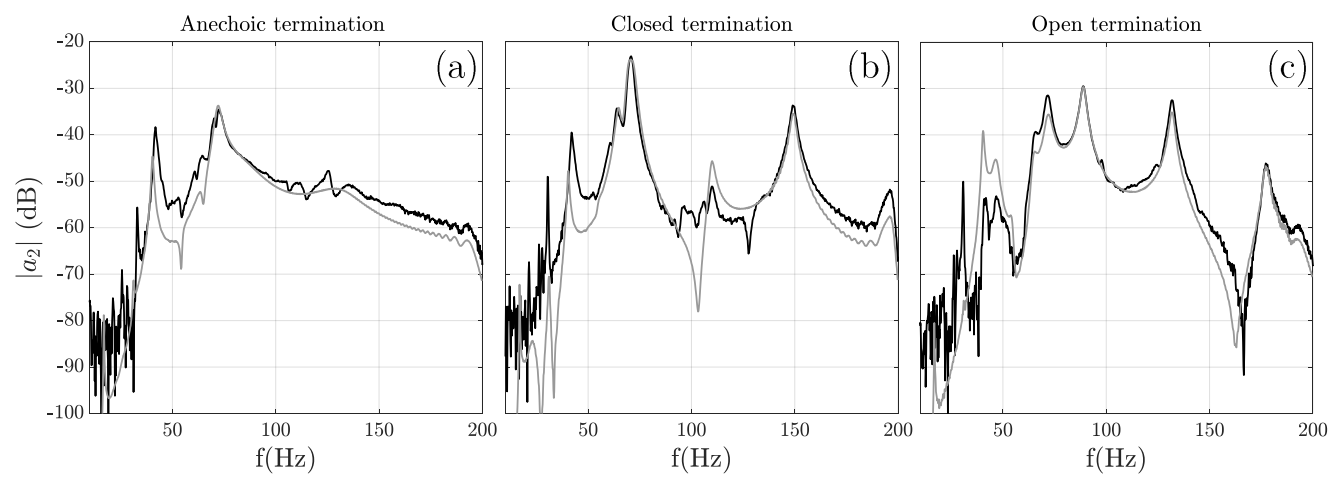

Figure 9: Measurement (black) and identification from the direct method (grey, Eq.10) of the acceleration at elbow 2 in the case of (a) an anechoic, (b) a closed and (c) an open termination.

This direct method can be very useful in industrial oil \& gas context. Indeed, by knowing in one hand, the mechanical mode shapes of a piping system from in situ measurements, and on the other hand, the sound pressure field from calculation tool (like simplified one-dimensional frequency models, Munjal [22]), it is possible to pre-estimate the critical vibration piping levels before the start-up of a reciprocating compressor.

Now, the regulated inverse identification procedure is being studied (eq.16). The $1^{\text {st }}$ line of the figure 10, corresponds to the estimated Sound Pressure Level (SPL) at elbow 2, based on the acceleration measurement in the 4 elbows. The (a), (b) and (c) graphs are still related to different pressure fields using respectively the anechoic, closed and open termination. 

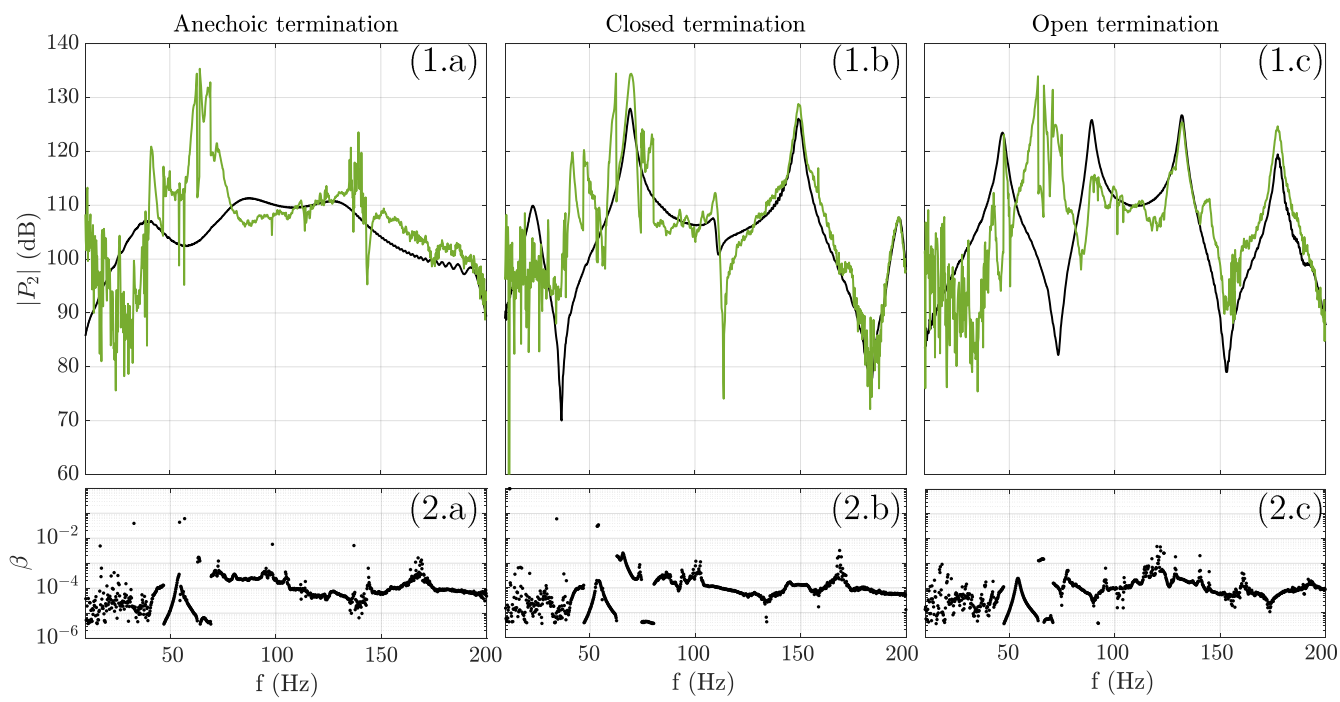

Figure 10: Experimental results of the inverse indentification of presures inside the pipe for (a) an anechoic, (b) a closed and (c) an open terminations. (1) measured (black) and identified (green) pressures at elbow 2; (2) regularization parameter $\beta$.

The pressure identification by the inverse method is relatively effective when sound pressure is important (e.g. on acoustic resonances). However, at other specific frequency bands, the identification is not satisfactory (e.g. around $70 \mathrm{~Hz}$ for graphs 1.a and 1.c). When the sound pressure field is too weak, the unbalanced forces are not sufficient to induce vibrations in the structure. In that case, the identification is affected by two phenomena:

1. noise measurement, for which the impact is reduced by the regularization method ensuring the consistency of the matrix inversion;

2. external mechanical solicitations, which are not taken into account in the theoretical model and are mainly induced by solid transmissions between the loudspeaker and the pipe. Note that in an industrial context, solid transmissions also exist between the reciprocating compressors and nearby pipes. Neglecting these solicitations is the roughest approximation, which could be interesting to overcome (e.g. by filtering it through a suitable signal processing method).

To verify the impact of these phenomena, the amplitudes of the unbalanced forces are compared with the error of pressure identification in Figure 
11. The graphs of the first line represent the three unbalanced forces associated with the three pairs of bends (1-2, 2-3, 3-4 - eq.5), for each termination. The red areas highlight the frequency bands where all the unbalanced forces are lower than the threshold of $5 \cdot 10^{-3} \mathrm{~N}$. The authors consider from these results that in such cases, the unbalanced forces no longer dominate the external solicitations. The graphs in the second line represent the deviation of the pressure amplitudes (SPL), averaged over the four elbows:

$$
d_{P}(f)=\frac{1}{4} \sum_{i=1}^{4}\left|\frac{\tilde{P}_{i}(f)-P_{i}(f)}{P_{i}(f)}\right| .
$$
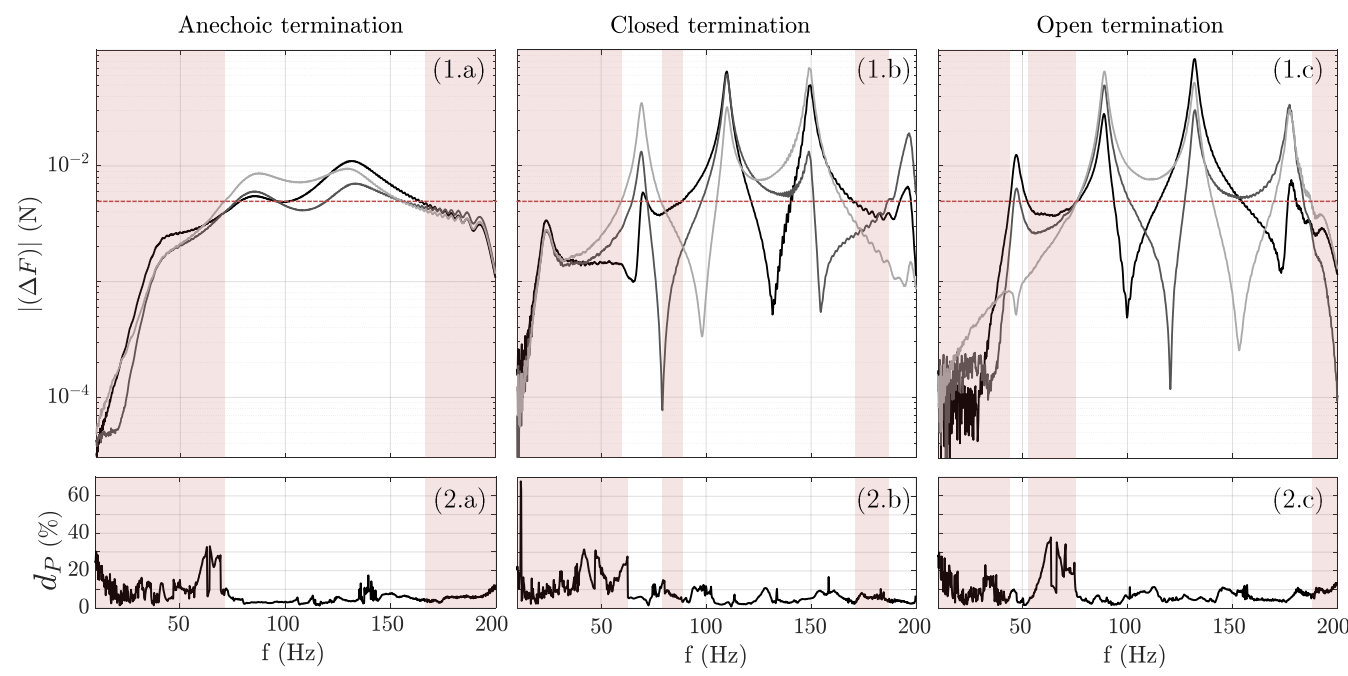

Figure 11: (1) amplitude of the unbalanced forces $\Delta F_{j}$ (eq.5) between each pair of elbow, from black $(j=1)$ to light grey $(j=3)$. (2) mean SPL relative deviation (eq.18). The red areas highlight the unbalanced forces threshold of $5.10^{-3} \mathrm{~N}$.

The aim is to show the limits of the inverse procedure when the unbalanced forces are low. The authors explain these errors by the presence of external solicitations due to solid transmission with the loudspeaker. These forces are neglected in the problem but are predominant when the unbalanced forces are close to zero. The threshold of $\Delta F=5 \cdot 10^{-3} \mathrm{~N}$, is an indicative but not generalizable threshold. It has been defined by the authors because the main error peaks $(\mathrm{dp}>10 \%)$ appear when the unbalanced forces drop below this threshold. 
For each configuration, significant errors are made at low frequencies $(<40$ $\mathrm{Hz}$ ), because on the one hand, the loudspeaker, whose cut-off frequency is 40 $\mathrm{Hz}$, is not efficient and on the other hand, the phase shift between the bends is too small due to the high wavelengths.

For resonant configurations, errors of approximately $10 \%$ also appear at anti-resonances (e.g. around $80 \mathrm{~Hz}, 100 \mathrm{~Hz}, 130 \mathrm{~Hz}$ with the closed termination and between $100 \mathrm{~Hz}$ and $110 \mathrm{~Hz}$ and around $150 \mathrm{~Hz}$ with the open termination).

Finally for each configuration, the main errors appear when the low unbalanced forces are combined with the high flexibility of the structure due to the presence of the mechanical modes in the X-Y plane (modes 3 at 40 $\mathrm{Hz}, 5$ at $65 \mathrm{~Hz}$ and 6 at $72 \mathrm{~Hz}$, as shown in the figure 5). In such cases the acceleration field measured in the bends is strongly affected by external solicitations (up to $50 \%$ error).

However, when the amplitude of the internal pressure is high enough to induce a vibration field dominating the external solicitations (here, $\Delta F>$ $5.10^{-3} \mathrm{~N}$ ), the relative deviation averaged over the 4 elbows remains small (generally $<5 \%$ ). This result is a key outcome of this work since it is obtained from only 4 acceleration measurements. Indeed, the interest of this procedure is mainly based on aspects such as fast and low cost measurements, because in Oil \& Gas vibration studies, the prolonged use of reciprocating compressors for diagnosis purpose, can be very cost-consuming.

To ensure the reliability of the method, the above analysis suggests that piping system have to be exposed to a more intense acoustical pressure field to increase the unbalanced forces. An acoustic source close to a reciprocating compressor is then chosen to approach the vibration conditions for the characterisation of piping systems.

\subsection{Experimental results: Internal pressure from an operational excitation induced by an electro-pneumatic source}

In this second experimental application, an electro-pneumatic source is used to generate a high amplitude pressure signal, very similar to a reciprocating compressor (Djuimo \& al [23]) . This source consists of a compression chamber from which the air is ejected into the pipe through a hole (fig.12(a)). 
The opening of this hole is modulated by the displacement of a disk (fig.12(b)) attached to a shaker.

This excitation method leads to the generation of high level pulsation waves inside the pipe ( $>140 \mathrm{~dB}$ SPL for the first harmonic), whose frequency components are controlled by the input shaker signal. As reciprocating compressors, this source generates large band noise due to the flow constriction through the disk, in addition to the high amplitude harmonic components which fundamental is set to $20 \mathrm{~Hz}$.

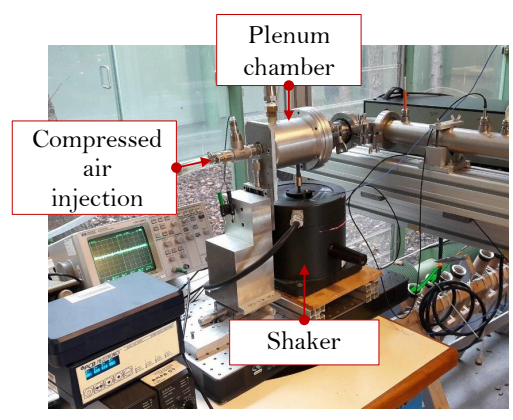

(a)

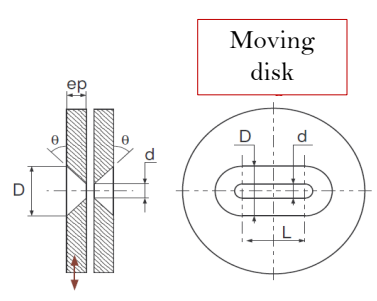

(b)

Figure 12: (a): high pressure electropneumatic source; (b) scheme of the moving disk connected to the shaker in order to modulate the plenum chamber aperture.

In graphs 13(a) and 13(b), the identified pressures (green) at elbows 2 and 3 , respectively, are compared with measurements (black), in the case of an open termination to let the pulsed flow out. Since the source is effective over the entire study frequency range $([5-200] \mathrm{Hz})$, the regularization parameter $\beta$ remains low even in low frequencies (figure 13(c)), which implies a high SNR.

First of all, except for the $1^{\text {st }}$ and the $4^{\text {th }}$ harmonics, a very good agreement between identification and measurement is observed for the harmonics amplitudes along the pipe, which are generated by the source. Moreover, acoustic resonances of the open ended pipe also appears (at $115 \mathrm{~Hz}$ and 165 $\mathrm{Hz}$ ), due to the wide band noise excitation of the source. Even if these resonances are much lower in amplitude than the harmonics (20 to $50 \mathrm{~dB}$ ), they are clearly captured by the inverse method. 

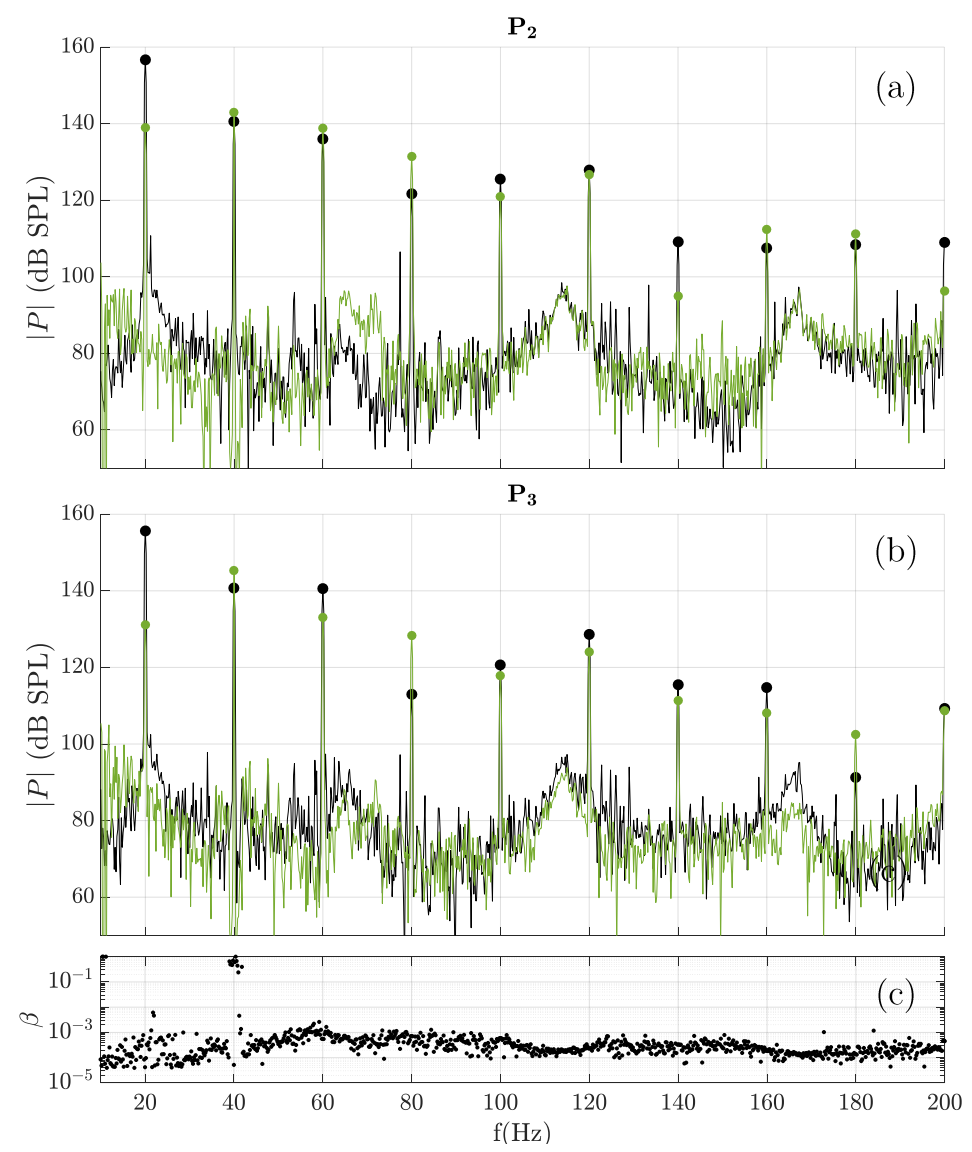

Figure 13: Application of the electro-pneumatic source with an open termination. (a) and (b) measured (black) and estimated (green, Eq.16) pressure amplitudes, respectively at elbows 2 and 3. (c): $\beta$ parameter used for the regularized solutions.

The large deviations at the $1^{\text {st }}$ and the $4^{\text {th }}$ harmonics can still be explained by external mechanical solicitations. Indeed, for the first harmonics, the unbalanced forces are very small, because of the the small acoustical phase shifts between the bends (at $20 \mathrm{~Hz}$, the wavelength is $17 \mathrm{~m}$ ). For the fourth harmonic, the frequency is close to the $3 r d$ mode $(72 \mathrm{~Hz})$, easily excited by solid transmission from the source. Few unexpected deviations remain ( $7^{\text {th }}$ harmonic at the elbow 2 or $9^{\text {th }}$ harmonic at elbow 3 ), meaning that the reduction of modelling complexities does not allow a high-fidelity reconstruction of the sound pressure. Despite these discrepancies, the level of identification is largely satisfactory to meet the need of troubleshooting studies for in-situ piping systems. Therefore, with few experimental precau- 
tions (the study area is far enough from a reciprocating compressor to limit structure borne excitations and the acoustic phase shift is sufficient between elbows), this inverse method fulfils its role as an efficient and non-intrusive diagnostic tool in the design of acoustic or vibratory treatments.

\section{Conclusion}

The developed model is driven by the measured modal characteristics in order to be used as an efficient diagnostic tool for in-situ troubleshooting applications in piping systems, that is non intrusive, simple, light and quick to use. To achieve that, the complexities has been reduced, i.e. the number of measurement points, the number of modes to be considered, the calculation time and the implementation procedure.

An inverse method is described to estimate the sound pressure levels within a U-shaped pipe line from mechanical acceleration measurements at the 4 elbows of the bend. The method is based on a modal representation of structural vibrations. From the direct solution of the problem, a 12 modes basis is validated for the identification of the structural forced response due to a broadband acoustic excitation. Then, a regularized inverse solution leads to the identification of the sound pressure. It involves a Tikhonov regularization, associated with an L-curve method to optimize the regularization.

The validity range of the procedure is evaluated numerically with an acoustic test signal, taking into account instabilities due to unavoidable measurement noise. Finally, two experimental applications of the inverse method are presented, involving three different kinds of pipe termination (open, closed and anechoic) and two kinds of acoustic excitation (loudspeaker and electropneumatic source).

The outcomes of this work lead to the following conclusions:

1. In the case where the vibrations induced by the pulsations of the fluid inside the pipe dominate the external vibrations, only a small number of vibration measurement points (4 points in the present U-shaped pipe) are required to accurately estimate the internal sound pressure.

2. The combination of Tikhonov regularization and L-curve methods leads to a quite stable solution, so that the inverse identification is mainly 
limited by geometric considerations. For example, the too short distance between two elbows relative to the relevant acoustic wave length, which implies that the unbalanced forces are too low to generate vibrations.

3. In the case of periodic acoustic excitation (close to reciprocating compressor signals), the method is able to discriminate the components of the acoustic source from those related to pipe resonances. It is then possible to detect in a non-intrusive way, the coincidence that may occur between them, resulting in significant vibrations.

From these analyses, this non intrusive diagnosic tool has attractive application potential to fast and light in-situ troubleshooting studies in piping systems.

Future additional improvement would be interesting to carry out in the domain of signal processing, to filter the external mechanical vibrations due to structure borne excitations and get even more precise results. However, it would certainly complicate the measurement setup.

\section{Acknowledgements}

The authors wish to thank Jean-Hugh Thomas from the LAUM and Dr Michael Newton from the University of Edinburgh, for valuable scientific discussions and assistance, as well as Eric Portier from the CTTM and Mathieu Secail-Geraud, Emmanuel Brasseur, Philippe Bequin and Julien Nicolas from the LAUM, for their support in setting up the experimental setup, Guillaume Raybaud and Antoine Schaeffer, ENSIM students, for the pre-dimensioning of the piping system and finally the ANRT for financing the project.

\section{References}

[1] Tison JD, Atkins KE, et al. The New Fifth Edition Of API 618 For Reciprocating Compressors-Which Pulsation And Vibration Control Philosophy Should You Use? In: Proceedings of the 30th turbomachinery symposium. Texas A\&M University. Turbomachinery Laboratories; 2001. . 
[2] Nakamura T, Kaneko S, Inada F, Kato M, Ishihara K, Nishihara T, et al. Flow-induced vibrations: classifications and lessons from practical experiences. Butterworth-Heinemann; 2013.

[3] Shkarayev S, Krashanitsa R, Tessler A. An inverse interpolation method utilizing in-flight strain measurements for determining loads and structural response of aerospace vehicles. NASA Langley Technical Report Server. 2001;

[4] Stanford B, Albertani R, Ifju P. Inverse methods to determine the aerodynamic forces on a membrane wing. In: 48th AIAA/ASME/ASCE/AHS/ASC Structures, Structural Dynamics, and Materials Conference; 2007. p. 1984.

[5] Carpenter T, Albertani R. Aerodynamic load estimation: Pressure distribution from virtual strain sensors for a pliant membrane wing. In: 54th AIAA/ASME/ASCE/AHS/ASC Structures, Structural Dynamics, and Materials Conference; 2013. p. 1917.

[6] Brown K, Brown J, Patil M, Devenport W. Inverse measurement of wall pressure field in flexible-wall wind tunnels using global wall deformation data. Experiments in Fluids. 2018;59(2):25.

[7] Engl HW, Kügler P. Nonlinear inverse problems: theoretical aspects and some industrial applications. In: Multidisciplinary methods for analysis optimization and control of complex systems. Springer; 2005. p. 3-47.

[8] Wiggert DC, Hatfield FJ, Stuckenbruck S. Analysis of liquid and structural transients in piping by the method of characteristics. Journal of fluids engineering. 1987;109(2):161-165.

[9] Lavooij CSW, Tijsseling AS. Fluid-structure interaction in liquid-filled piping systems. Journal of fluids and structures. 1991;5(5):573-595.

[10] Zhang L, Tijsseling SA, Vardy EA. FSI analysis of liquid-filled pipes. Journal of sound and vibration. 1999;224(1):69-99.

[11] Maess M, Gaul L. Substructuring and model reduction of pipe components interacting with acoustic fluids. Mechanical systems and signal processing. 2006;20(1):45-64. 
[12] Herrmann J, Maess M, Gaul L. Substructuring including interface reduction for the efficient vibro-acoustic simulation of fluid-filled piping systems. Mechanical systems and signal processing. 2010;24(1):153-163.

[13] Li Sj, Liu Gm, Kong Wt. Vibration analysis of pipes conveying fluid by transfer matrix method. Nuclear Engineering and Design. 2014;266:7888.

[14] Blodgett L. Advanced pulsatrion/vibration analysis - PCRC Short course $\mathrm{N}^{\circ} 93 / \mathrm{A} / 4$. In: International Reciprocating Machinery Conference - Pipeline \& Compressor Research Council; 1993. .

[15] Katz VJ. The history of Stokes' theorem. Mathematics Magazine. 1979;52(3):146-156.

[16] Idier J, editor. Bayesian Approach to Inverse Problems. ISTE Ltd and John Wiley \& Sons Inc; 2008.

[17] Tikhonov AN. Solution of incorrectly formulated problems and the regularization method. Soviet Math Dokl. 1963;151:1035-1038.

[18] Hansen PC. Analysis of discrete ill-posed problems by means of the L-curve. SIAM review. 1992;34(4):561-580.

[19] Dalmont JP, Portier E. Optimisation of anechoic duct termination using line theory. Applied Acoustics. 2017;117:141-144.

[20] Ege K, Boutillon X, David B. High-resolution modal analysis. Journal of Sound and Vibration. 2009;325(4-5):852-869.

[21] Paiva GO, Ablitzer F, Gautier F, dos Santos JMC. The Roving WireBreaking Technique: a low cost mobility measurement procedure for string musical instruments. Applied Acoustics. 2018;139:140-148.

[22] Munjal ML. Acoustics of Ducts and Mufflers With Application to Exhaust and Ventilation System Design. A Wiley-Interscience publication. Wiley; 1987. Available from: https://books.google.fr/books?id= Z-s50vk-U68C.

[23] Djuimo CK, Dalmont JP, Béquin P, Gilbert J, Dupuis PE, Hirschberg A. Source électropneumatique : étude des écoulements subsonique et sonique. In: CFA/VISHNO 2016. Le Mans, France; 2016. . 


\section{Appendices}

\section{A. L-curve method (Hansen [18])}

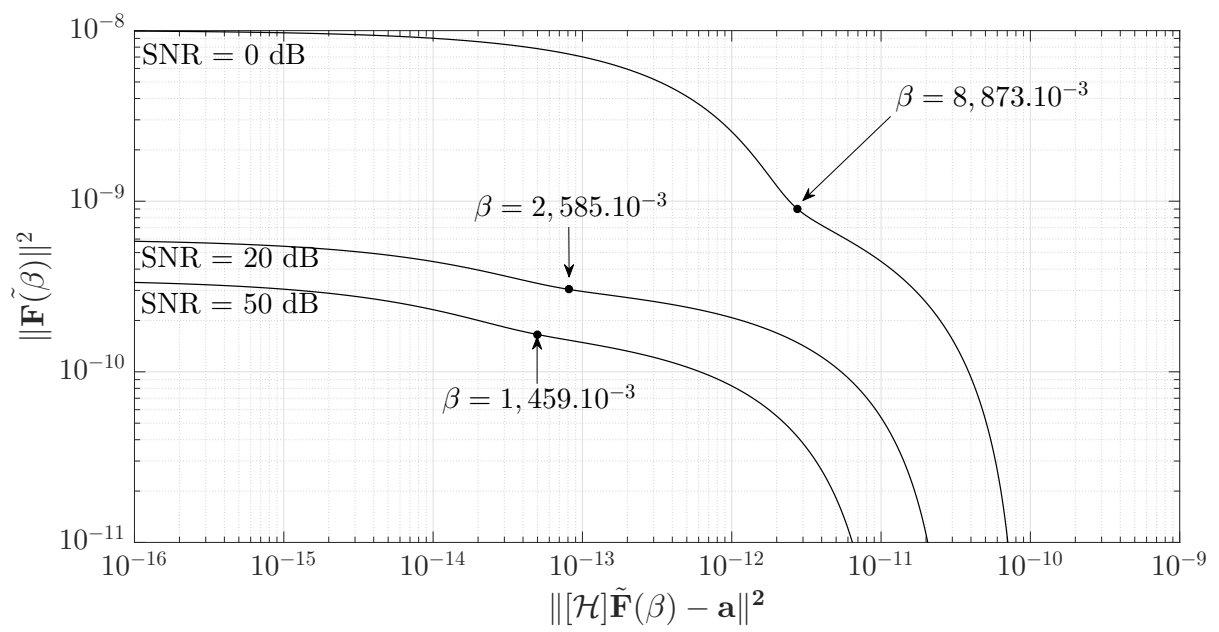

Figure A.1: L-curve at $50 \mathrm{~Hz}$ for SNRs $=[50,20,0] \mathrm{dB}$, obtained using white Gaussian noise as input signals in elbows (fig.7), with $\tilde{\mathbf{F}}$ coming from equation 16. The optimal $\beta$ argument is obtained at the knees of the L-curve functions.

The figure A.1 represents the 2-norm of the function $\tilde{\mathbf{F}}$ (eq.16) plotted in regard to the 2 -norm of the argument $\mathcal{H}] \tilde{\mathbf{F}}-\mathbf{a} \mid$ when $\beta$ varies. The Lcurve method is an optimal regularization technique based on the detection of a $\beta$ value minimizing the error between $\mathbf{F}$ and $[\mathcal{H}]^{-1} \mathbf{a}(\omega)$ (eq.12). The function shapes an L-curve with the optimal value of $\beta$ located at the point of maximum inflection of its curvature. For the detection of this inflection point, the choice was made to use a simple method based on the detection of the zero value of the second derivative of $\tilde{\mathbf{F}}(\mathcal{H}] \tilde{\mathbf{F}}-\mathbf{a} \mid)$.

\section{B. the quasi anechoic termination}

The quasi anechoic termination is based on the progressive adaptation of acoustic external impedance, using a specific distribution of side holes (fig.B.1(a)). The reflection coefficient has been experimentally characterized, 
using a pipe inlet impedance measurement bench (Dalmont \& Portier [19]). This reflection coefficient is plotted in figure B.1(b).

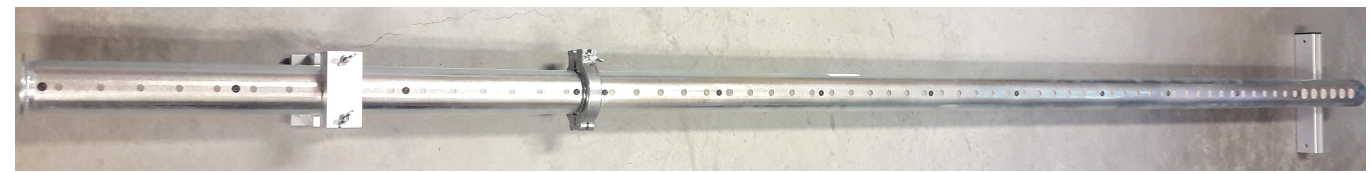

(a)

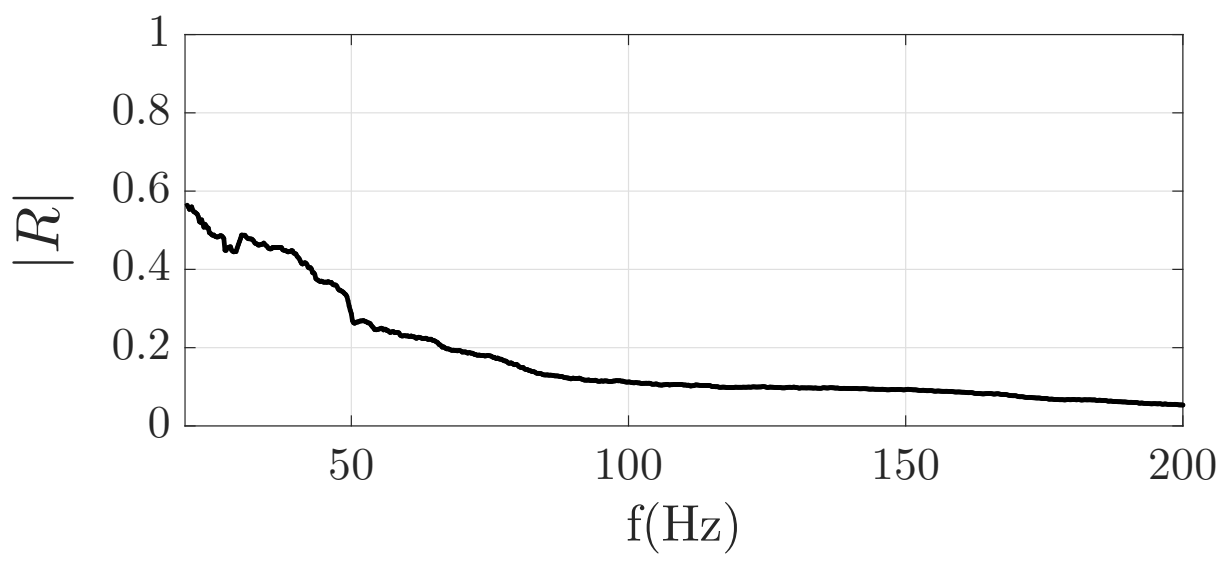

(b)

Figure B.1: (a) quasi anechoic termination with side holes ; (b) measured reflection coefficient at the entrance of the quasi anechoic termination. 